\title{
Construction and Application of Innovative Practice Platform Based on "Explorer"
}

\author{
Zijiang Yang ${ }^{1,}$, , Fei Wang ${ }^{2, ~ b}$, Xiangning Pan ${ }^{1, ~ c}$, Weifeng Tang ${ }^{1, d}$ \\ ${ }^{1}$ Naval Aviation University, Yantai 264001, China; \\ ${ }^{2}$ QiLu University of Technology (Shandong Academy of Sciences), Jinan 250353, China. \\ ayzjkgjj@163.com, b448167701@qq.com, c531519045@qq.com, dtanghuotwf@163.com
}

\begin{abstract}
By introducing the "Explorer" innovation platform, to make up for lack of original innovation components, openness and enhance the mechanical engineering innovation practice. In view of the limitations of "Explorer", 3D printing technology, mechanical design software, mainstream electronic modules, and standard parts and self-processing parts are applied. Based on the "Explorer", a new mechanical innovation practice platform is built to break the limitation of parts and structures, expand parts design space and optimize hardware configuration. Practice has proved that the new mechanical innovation practice platform can meet the needs of students more easily and achieve goals more easily. It is beneficial to release students' innovative thinking, improve students' practical ability and speed up the cultivation of students' engineering literacy.
\end{abstract}

Keywords: Explorer, Machinery, Innovation, Engineering, practice.

\section{Introduction}

Military engineering colleges and Engineering Majors in the military academy shoulder the mission of cultivating engineering innovative talents for the army[1-3].Mechanical innovation practice has a good effect in cultivating innovative talents in engineering and technology fields. It not only helps to cultivate students' innovative spirit, innovation consciousness, and innovation ability, but also accelerates the cultivation of students' engineering literacy. At present, it has been recognized and pushed by military academies and even local colleges [4-8].

To carry out mechanical innovation and practice activities, the innovative components (platforms) needed for production are very important. There are many kinds of modular innovative components (platforms) used by universities to carry out innovative practice activities, which can be divided into closed platforms, semi open platforms and open platforms [9-10].In the past, the closed modular innovation component had many shortcomings and was out of the mainstream. For this reason, our school introduced a group of explorers' innovation platform, or short term "Explorer". This product adopts metal structure parts with high strength and stiffness; all electronic modules are designed with open design. It can be butted seamlessly with all kinds of mainstream electronic modules and has good interactivity. The programming software uses Arduino IDE, Chinese graphical interface, easy to learn and easy to operate, and can be converted into $C$ language. Based on this platform, we should break the original restrictions and expand. It can be closer to the engineering practice, adapt to the requirements of the production, and meet the needs of the students, not only to enhance the students' awareness of innovation, improve the creative ability of the students, but also speed up the cultivation of students' engineering accomplishment.

\section{Application Status of "Explorer"}

\subsection{Aasic components of the "Explorer" Parts}

The "Explorer" combines many advanced technologies, such as mechanical, electronic, sensor, computer software and hardware, control, artificial intelligence and modeling technology, and its parts are versatile, practical and engineering[11].Its basic composition is shown in Figure 1. 


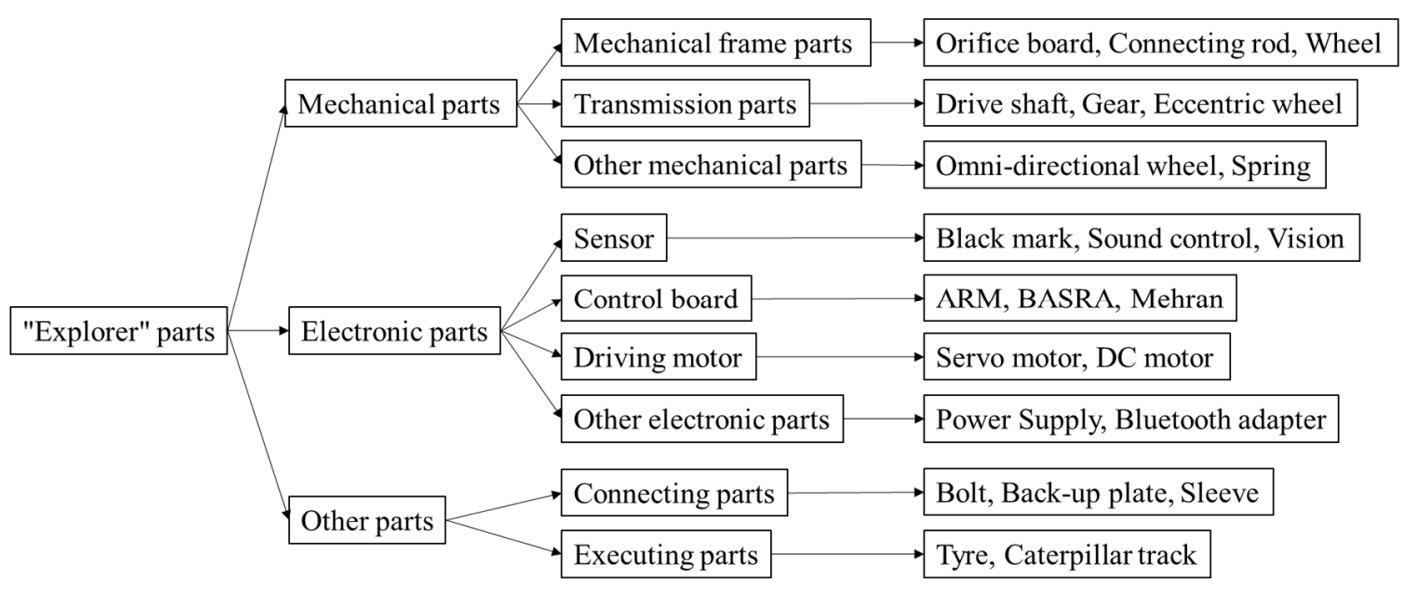

Fig 1. The basic components of the "Explorer" parts

\subsection{Application Case of "Explorer"}

(1) Case 1: Tracked all terrain robot

The robot is designed and manufactured under the framework of "Explorer", and the whole machine is fully automatic design. Full application of "Explorer" structure design software and Arduino programming software; through the BASRA, it has the functions of automatic tracking, automatic correction, and automatic obstacle avoidance and so on. The specific structural form is shown in Figure 2. The works won the two prize in the 2017 RoboForm competition.

(2)Case 2: Automatic sweeping robot

The design and production of the automatic sweeping robot are also completed under the framework of "Explorer". The design and production process of the whole machine is similar to the case 1 .The robot can realize automatic walking, automatic obstacle avoidance and automatic sweeping. The specific structure is shown in Figure 3.Also in the "exploration road" innovative practice, we won the two prize.

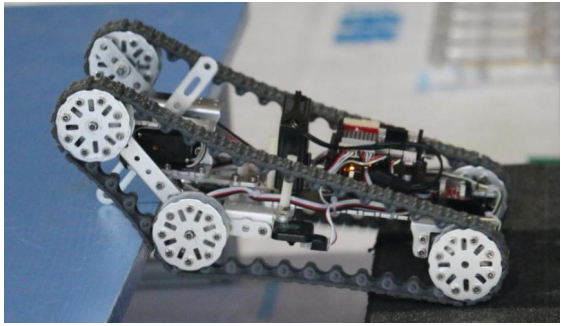

Fig 2. Tracked all terrain robot

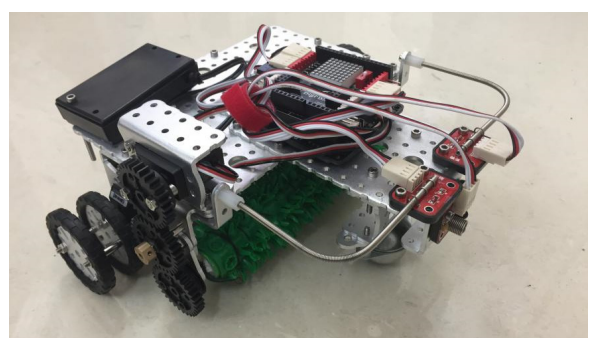

Fig 3. Automatic sweeping robot

\subsection{Case Analysis of "Explorer"}

The above two cases prove the advantages of "Explorer" in the design and production of mechanical innovation practice. But it also exposes some insufficiencies, like the following.

(1) The structure of "Explorer" is solidified and cannot form complex parts.

(2) The size of the mechanical parts is small and is not suitable for making larger size works.

(3) Its structural design software only has parts calling function, lacking standard parts library and non-standard parts design function.

(4) The speed and torque of the DC motors and servo motors are smaller, so they are helpless when facing the demand of high speed and large torque.

\section{Construction of New Platform for Mechanical Innovation Practice}

On the basis of "Explorer", under the principle of retaining advantages and making up for defects, a new practical platform for mechanical innovation is constructed so as to reach more close to the actual project and to meet the needs of production. 
(1) Use 3D printing technology to break the structural limitations of parts.

3D printing technology is also called additive manufacturing technology. Compared with traditional manufacturing methods, its advantage lies in its short manufacturing cycle and the fact that it's forming is not limited by the complexity of parts [12]. The application of 3D printing technology can break the structural limitations of components and complete the manufacture of complex structural parts.

(2) Introduce standard parts and self-processing parts to overcome the limitation of parts size.

As a category of mechanical parts, standard parts have already achieved standardized production. The commonly used standard parts in mechanical innovation practice are: European standard aluminum profiles, bearings, bolts and so on. For some standard parts, micro machining, micro dressing and simple machine tool processing can be done. Through these works, we can break the limitation of parts size of "Explorer".

(3) Use mainstream design software to expand parts design space.

SolidWorks is a powerful 3D CAD software, which can easily complete the creation of 3D model, motion simulation, data management and so on under the framework of [13,14]. By applying its 3D modeling and motion simulation functions, the initial design can be modified and adjusted, and the design flexibility is great.

MD 3D tools is a professional mechanical design software, whose feature is the establishment of a comprehensive and practical standard parts library and parts library. Students can easily retrieve, invoke, and directly generate 3D models, saving energy and time for consulting related standards manual and drawing relevant standard parts.

(4) Introduce mainstream electronic modules to optimize hardware configuration.

The motor speed of the "Explorer" parts is below 80, and the torque is below 5. Sometimes it is necessary to introduce high speed or large torque motor. However, the main boards such as BASRA and ARM cannot drive them directly. At this point, L286N motor drive module and brushless electric adjustment can play a role. For simple control tasks, by introducing some entry-level master boards, such as "51 single chip microcomputer", etc., the cost of parts can be cut down.

\section{Application Effect of New Platform of Mechanical Innovation Practice}

Through practice tests, the new mechanical innovation practice platform has obvious advantages, and it can well meet the needs of students participating in various mechanical innovation practice activities and competitions.

\subsection{Application Case of New Platform for Mechanical Innovation Practice}

(1) Case 1: Variable all-round moving crane

The specific structure of the crane is shown in Figure 4 (a), and its design and production are completed under the new mechanical innovation practice platform. The design process is completed with the aid of SolidWorks and MD 3D design tools, as shown in Figure 4 (b); the control part (main board, remote control, etc.), the part connection and programming use "Explorer"; the material of the frame part is selected for European standard 1515 aluminum profile; the power part uses the higher output torque. The flow deceleration motor and L298N motor drive module are adopted, and the traveling part adopts the Meconium wheel.

The new platform for mechanical innovation practice has more advantages than "Explorer" Therefore, it won the first prize in the 2017 China mechanical engineering innovation and originality competition. 


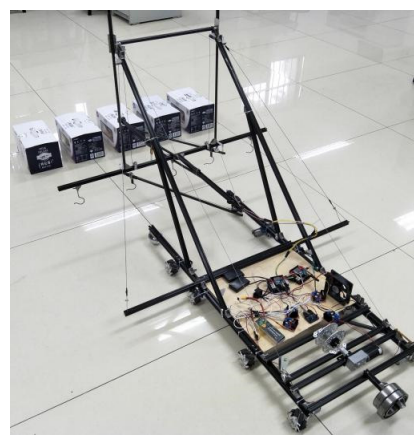

(a) Practicality picture

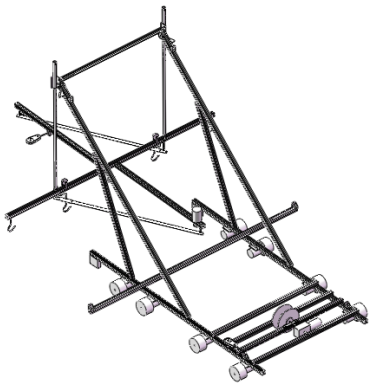

(b) 3D Model

Fig 4. Variable all-round moving crane

(2) Case 2: HJ-1 antagonism robot

The specific structure of the robot is shown in Figure 5. In addition to the integration of the "Explorer" machine parts in the whole frame, the power part uses brushless motor and brushless electric adjustment, the main control board uses the PC2 programming card of the car, and the remote control function is realized by the $\mathrm{RC} 3 \mathrm{~S}$ remote control and the $\mathrm{R} 4 \mathrm{EH}-\mathrm{H}$ receiver.

Because of the new platform, the robot won the first prize of the 2017 China engineering robot contest, and its technical report has been included by the organizing committee.

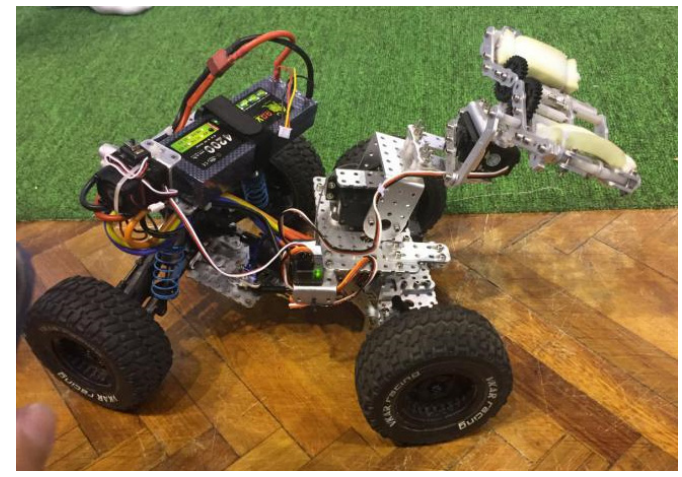

Fig 5. HJ-1 antagonism robot

\subsection{Application Case Analysis of the New Platform of Mechanical Innovation Practice}

The whole process of the design and production of the above two works strongly proves the practicality, universality and engineering of the new mechanical innovation practice platform, which can meet the needs of various kinds of mechanical innovation practices and competitions.

\section{Summary}

It has been proved that the "Explorer" has made a qualitative improvement on the structure strength, stiffness, hardware interface and programming software, but there are still a lot of shortcomings in itself. The new practice platform of mechanical innovation makes up for the shortage of "Explorer" itself, breaks the limitation of the structure and size of parts and components, expands the design space of parts and components, and optimizes the hardware configuration. The new platform can adapt to more production needs and play a role in more mechanical innovation practices and competitions.

\section{References}

[1]. Shipping Chen. On the implementation of innovative education in military engineering colleges, [J]. Journal of higher education research, 2003, 26 (3): 26-28. 
[2]. Gang Ling. On the cultivation and development of the army's innovative science and technology talents [J]. Journal of Nanjing Political College, 2012, 28 (3): 118-120.

[3]. Wei Chen, Bo Sun, Songhua Cao. Reflections on the cultivation of innovative military talents in military academies [J]. Science and education Whenua, 2010 (4): 44-44.

[4]. Juju Ying, Yuan Chen, Jibe Liu, teal. Analysis on the current situation of military university students' competition activities and the cultivation of innovative talents. [J]. education and teaching forum, 2016 (41): 102-103.

[5]. Zheng Yun, Alan Wang, Liaoyang Tang. Research and Practice on the training model of mechanical engineering extended talents based on innovation competition [J]. Journal of higher education research, 2010, 33 (3): 95-98.

[6]. Hai Guan, Young Li. The training mode of mechanical innovation talents based on ability training. [J]. Journal of Dalian University, 2009, 30 (6): 123-125.

[7]. Haring Zheng, Pan Xu, Pealing Zhang, teal. Investigation and analysis of the management status of extracurricular creative practice activities for undergraduate students of Military Academy Taking the University of National Defense Science and technology as an example of [J]. Higher education research journal, 2015 (3): 62-66.

[8]. Don sheng Zhang, Xiao Jun Wang, Zheng Zhang, teal. Research on the operation mechanism of local university students' mechanical innovation design competition [J]. China modern educational equipment, 2016 (7): 89-92.

[9]. Youngman Wang, Jian Hong Ge, Bingaman Wang. Research and exploration of robotics innovation and opening laboratory construction [J]. Laboratory, 2009, 28 (11): 4-5.

[10]. Zigong Zhu, Haile Xu, Longboat Zhu, teal. Design and implementation of innovation and practice project of mechanical and electrical specialty [D].2009.

[11]. Hailing Ge, Globin Chen. Experimental teaching reform of robotics curriculum based on Explorer platform [J]. Development and innovation of electromechanical products, 2016 (4): 121-122.

[12]. Xuan Zhang, Siri Tang, Dengue Zhao, and other.3D printing technology research status and key technologies [J]. Material engineering, 2016, 44 (2): 122-128.

[13]. Winching CEng. Design and application of mechanical parts based on SolidWorksd [J]. Manufacturing automation, 2012 (4): 135-137.

[14]. Xiaoping Yu, Fan-tan Lain, Xiao Hong Wang, teal. The mechanical design of the SolidWorks based inspection robot and the simulation of the obstacle movement [J]. Mechanical design and manufacture, 2010 (8): 180-182. 